\title{
Expérience vécue chez un chef étoilé et achat de produits locaux par les clients
}

\section{Marielle Salvador-Pérignon}

\section{(2) OpenEdition}

1 Journals

Édition électronique

URL : http://journals.openedition.org/tourisme/79

DOI : $10.4000 /$ tourisme. 79

ISSN : 2492-7503

Éditeur

Éditions touristiques européennes

\section{Édition imprimée}

Date de publication : 1 décembre 2013

Pagination : 19-32

ISSN : 2109-5671

\section{Référence électronique}

Marielle Salvador-Pérignon, «Expérience vécue chez un chef étoilé et achat de produits locaux par les clients », Mondes du Tourisme [En ligne], 8 | 2013, mis en ligne le 01 juin 2015, consulté le 01 mai 2019. URL : http://journals.openedition.org/tourisme/79 ; DOI : 10.4000/tourisme.79

\section{cc) (†) $\ominus$}

Mondes du tourisme est mis à disposition selon les termes de la licence Creative Commons Attribution - Pas d'Utilisation Commerciale - Pas de Modification 4.0 International. 


\title{
Expérience vécue chez un chef étoilé et achat de produits locaux par les clients
}

\author{
Maruelle SalVADOR-PÉrignon \\ [msalvadon@inseec.com] \\ Enseignant chercheur en sciences de gestion, Inseec Alpes-Savoie \\ Qualifié aux fonctions de maître de conférences, associé à l'Irege, Université de Savoie
}

Résumé. La plupart des chefs étoilés mettent à l'honneur les produits locaux. Ils contribuent ainsi à mieux faire connaître les produits emblématiques de leurs régions auprès de leurs clients. Si la plupart des études s'intéressent à l'offre, nous nous intéressons ici à la demande et cherchons à mieux comprendre à travers une étude exploratoire le rôle que peuvent jouer les chefs étoilés dans l'achat de produits locaux par leurs clients à travers l'expérience gastronomique vécue par ces derniers. Les chefs étoilés seraient alors, non plus seulement des ambassadeurs de leurs régions, mais de véritables prescripteurs des producteurs de produits locaux.

Abstract. Most of chefs highlight local products through their cooking. They help to reveal to their customers their local iconic products. While most studies focus on offer, we are interested in demand. Through an exploratory study, we try to understand the role of the chefs in the purchase of local products by their customers through a gastronomic experience. The chefs would be not only ambassadors for their regions, but real prescribers for local producers. 
$\mathbf{L}$ e développement, depuis quelques années, des émissions de télévision, blogs, livres ou encore des coffrets de séjours gastronomiques illustre le regain d'intérêt pour la cuisine, et l'alimentation en général. Les sociologues de l'alimentation (Corbeau et Poulain, 200I) expliquent la quête de sens et de valeurs exprimée à travers les produits alimentaires par le paradoxe auquel sont confrontés les individus, tout du moins dans notre hémisphère : n'ayant plus désormais le souci vital de trouver leur nourriture, les voici confrontés à celui de choisir ce qu'ils désirent manger. Face aux crises alimentaires de ces dernières décennies, chacun cherche aujourd'hui une certaine authenticité dans son alimentation, un retour à des valeurs dans lesquelles il se reconnaisse et qui soient facteurs de réassurance. Or les produits locaux, parce qu'ils sont distribués et consommés à proximité de leur lieu de production, sont parmi les produits susceptibles de répondre à leurs attentes.

On retrouve aujourd'hui ces produits sur les plus grandes tables, ce qui contribue à rendre mémorable l'expérience vécue dans un établissement "étoilé” (c'est-à-dire distingué par une ou plusieurs étoiles par le guide Michelin). Michel Bras et Marc Veyrat, par exemple, qui ont à cour de faire découvrir à leurs clients les produits les plus emblématiques du lieu, sont ainsi devenus, à travers leur cuisine, de véritables ambassadeurs de leur territoire (l'Aubrac et la Savoie, respectivement).

Dans un restaurant étoilé, la personnalité du chef et ses engagements dans la valorisation des produits issus de l'économie locale deviennent partie intégrante de l'expérience vécue. Le chef crée alors le haut lieu touristique (Bonnain-Dulon et Brochot, 2008). Dans ce contexte, s'il joue un rôle sur l'attractivité du territoire, tant auprès des touristes que des résidents du lieu, il pourrait également agir en tant que prescripteur et favoriser ainsi l'achat de produits locaux. Nous nous interrogeons donc sur le rôle effectif des chefs étoilés dans l'achat de produits locaux à travers l'expérience de gastronomie vécue par leurs clients.

La première partie de cet article s'attache à présenter les concepts retenus pour cette recherche afin d'analyser les liens existants entre les produits locaux, les éléments constitutifs d'une expérience de consommation dans le contexte du restaurant étoilé et le rôle de prescripteur qui pourrait être celui du chef étoilé. Dans une seconde partie, nous présentons la méthodologie de l'étude puis, à travers l'analyse des récits collectés, nous exposons les résultats qui nous amènent à nous interroger sur les raisons qui, selon nous, freinent l'influence qu'un chef étoilé pourrait avoir sur le comportement d'achat de ses clients de produits locaux chez les producteurs consécutivement à l'expérience gastronomique vécue.

\section{PRÉSENCE DES PRODUITS \\ LOCAUX DANS LES \\ RESTAURANTS ÉTOILÉS :}

UNE FORME DE PRESCRIPTION ?

Les récentes recherches en marketing, en sociologie de l'alimentation et en anthropologie ont permis de définir avec précision les caractéristiques d'un produit local et, ainsi, de le distinguer notamment du produit du terroir.

\section{Le produit local :}

\section{précisions terminologiques}

Le produit local est défini comme étant un produit distribué et consommé à proximité de son lieu de production, évalué entre quatrevingt et cent kilomètres (Merle et Piotrowski, 2012). Il se caractérise donc par son attachement à un lieu géographique et par l'existence de relations proches entre le producteur et le consommateur (Vitterso et Amilien, 201 I).

Il se distingue du produit du terroir dans le sens où ce dernier revêt, en plus d'une origine géographique, une dimension spécifique liée au savoir-faire et à l'histoire de la recette (Aurier, Fort et Sirieix, 2005) qui en fait parfois un produit protégé par une labellisation de type AOP (appellation d'origine protégée) ou AOC (appellation d'origine contrôlée) et pour lequel le circuit peut être plus long (Amilien, 2005).

Dans un contexte sociétal d'inquiétude face à des "objets comestibles non identifiés” (Fischler, 1990) et à la multiplication des crises agroalimentaires, les produits attachés à 
un lieu inspirent confiance et rassurent. En effet, la proximité du lieu de production et du producteur favorise une meilleure représentation $\mathrm{du}$ rôle de ce dernier dans la fabrication du produit (Merle et Piotrowski, 20।2). De même, les relations directes entre producteur et consommateurs ont un rôle sur la confiance dans la perception de la qualité des produits locaux (Prigent-Simonin et HéraultFournier, 2005).

Le circuit court, caractéristique du produit local, recouvre différentes formes de distribution. La vente au bord des routes et sur les marchés constitue un premier degré d'interactions entre le producteur et le consommateur (Fiamor, 20 I I). La vente dans des points de distribution collectifs, comme ceux développés par les Amap (associations pour le maintien d'une agriculture paysanne), et la vente à la ferme constituent un second degré d'interactions caractérisé par une réelle proximité avec le producteur. En effet, lors d'une vente à la ferme, le consommateur pénètre sur le lieu de production, le plus souvent dans une annexe de la maison, et peut être amené à voir les vaches ou les chèvres, par exemple, ainsi que le lieu de transformation du lait en fromage. Mais, quel que soit le niveau d'interaction, ce comportement d'achat traduit d'une part, l'adhésion du consommateur à une autre conception de l'agriculture et de la production et, d'autre part, sa participation active à sa valorisation (Fiamor, 20I I). Des engagements que certains chefs étoilés mettent claire- ment en avant aujourd'hui, participant ainsi à la valorisation et à la reconnaissance du travail des producteurs locaux. Le produit local devient alors un élément de l'expérience de repas gastronomique.

\section{Expérience de repas chez un chef étoilé et produits locaux}

Un produit local trouve un écho favorable auprès des consommateurs et se retrouve aussi bien dans les assiettes de tous les jours que sur les plus grandes tables. Dans la mesure où ce type d'expérience reste encore exceptionnel pour la majorité des individus, il constitue alors un élément de l'expérience mémorable vécue par le client. Le ravissement et la surprise que peuvent provoquer un plat sont des éléments qui permettent de caractériser le repas dans un restaurant étoilé comme une expérience de consommation exceptionnelle au sens d'Arnould et Price (1993). Dans ce contexte, et à la lumière du concept de la prescription, le chef étoilé, par la cuisine qu'il propose à ses clients, pourrait être prescripteur d'achat de produits locaux auprès de producteurs de sa région.

Le chef d'un grand restaurant crée et développe un phénomène expérientiel pour son client. Son offre est à la fois imprégnée de rituels, au travers d'actes et de paroles codifiés, mais également de sensations (Hetzel, 2004). Ces deux composantes font du repas gastronomique une expérience de consommation qualifiée d'expérientielle, car elle est source de plaisir, facteur de sens et mémo- rable pour le client (Filser, 2008). Du point de vue du comportement du consommateur, l'expérience d'un repas gastronomique est subjective et intègre une dimension émotionnelle et sensible (Carù et Cova, 2006).

Notre recherche se positionne davantage sur les déterminants d'une expérience de consommation que sur la valeur de l'expérience vécue (Holbrook, 1999 ; Aurier, Fort et Sirieix, 2005) ou que sur la mesure de l'expérience vécue comme résultat de l'interaction d'un lieu et d'un objet (Mencarelli, 2008).

Les déterminants d'une expérience de consommation ont été mis en relief par Roederer (2008) au travers de trois dimensions qui trouvent à s'appliquer dans le cas d'un repas dans un grand restaurant.

La première est une dimension hédonico-sensorielle portant sur le plaisir (ou déplaisir) retiré de l'expérience. Dans un contexte gastronomique, cette dimension pourrait se traduire par le contenu émotionnel, d'une part, et par la nourriture consommée, d'autre part. En effet, Bayet-Robert (20I I) distingue l'expérience de repas gastronomique d'abord selon son contenu émotionnel, l'évocation d'une expérience passant par l'intensité de celui-ci, qu'il soit positif ou négatif, suscité chez l'individu (Filser, 2002). Dès lors, selon Bayet-Robert, l'expérience d'un repas chez un chef étoilé est bien une expérience extraordinaire au sens d'Arnould et Price (1993), car l'on y retrouve les composantes du flow psychologique (Csíkszentmihályi, 1990)(1), qui correspond à un état 
mental optimal de concentration intense dans lequel l'individu se sent complètement absorbé par ce qu'il fait (Kréziak et Frochot, 20 I I). Le souvenir d'une telle expérience reste dans les esprits en tant qu'événement exceptionnel, hors du commun. Le niveau de ravissement peut tenir aussi à la surprise provoquée et entraîner une satisfaction extrême (Vanhamme, 2008). L'expérience gastronomique se distingue également par l'assiette ou la "nourriture consommée” (Bayet-Robert, 20II), dont le contenu passe par l'aspect esthétique. Les recherches sur ce point s'accordent sur le fait que la présentation d'un plat est un des éléments favorisant une expérience positive (Walter 20I I, cité par Bayer-Robert 20 I I). Dans un contexte de concurrence accrue, cette nouveauté permet aux établissements étoilés de se différencier ${ }^{(2)}$. Pour se démarquer, ceuxci doivent proposer des plats inédits composés d'ingrédients uniques que l'on ne pourra pas retrouver sur une autre table (Bonnain-Dulon et Brochot, 2008). Ces ingrédients peuvent être des fleurs qui participent à l'esthétique de l'assiette, une herbe particulière du lieu (chénopode, aspérule, polypode...), un légume dit "oublié" (panais, topinambours...) préparé de façon inédite ou un produit local sublimé (les fleurs de courgettes en Provence, la polenta en Savoie, etc.).

La deuxième est une dimension socioculturelle de l'expérience soulignant le sens donné à l'expérience vécue par l'individu au travers de son récit; celui-ci étant "une forme de jugement social nécessairement lié à une culture donnée. La dimension de l'expérience qui expose ou qui permet au sujet de se soumettre en quelque sorte au regard social, formé des valeurs et des croyances propres à un contexte social donné" (Roederer, 2008). La tenue vestimentaire est à ce titre un point incontournable dans la réalisation de l'expérience gastronomique (Bayet-Robert, 20II). Les interactions avec le personnel en salle, voire le chef, entrent également dans cette dimension. Les échanges avec le sommelier ou le maître d'hôtel participent à la cocréation de l'expérience par le client lui-même, cette participation se révélant importante pour vivre une expérience extraordinaire (Amould et Price, 1993).

Enfin, une troisième dimension de l'expérience est une dimension temporelle, puisque l'individu contrôle la durée de son expérience vécue. Dans le contexte d'un repas gastronomique, cette dimension se traduit aux travers des plats, qui se succèdent selon un ordre établi, mais également des rites et actes codifiés qui entourent le repas (Hetzel, 2004) et qui laissent, en définitive, peu de maîtrise de l'expérience que vit l'individu.

Pour les chefs étoilés, mieux comprendre et connaître les dimensions de l'expérience de repas gastronomique permet de créer des contextes expérientiels adéquats. Si l'exaltation des sens visuels (via l'esthétique de l'assiette, de l'odorat, du toucher) ou du goût (via les sensations des mets portés à la bouche) constitue une part importante de l'expérience de repas gastronomique, les interactions avec le personnel, la rencontre avec le chef sont également des éléments indispensables à la production d'un contexte expérientiel. De même, les éléments liés à l'art de la table font partie de l'offre, tout autant que la décoration de la salle. Enfin, le lieu même du restaurant, son environnement extérieur sont importants. Par exemple, se rendre chez Michel Bras dans l'Aubrac tend à contribuer à la création d'un contexte expérientiel.

L'offre d'expérience ne se limite pas à la seule créativité de la cuisine du chef ; elle est aussi constituée par le décor dans son ensemble (arts de la table, atmosphère de la salle, lieu de situation de l'établissement) et par le récit ou l'histoire sur le produit que le chef décide de mettre en avant à travers la présentation par le personnel en salle ou la carte. On retrouve ainsi les trois éléments de l'offre d'expérience mis en relief par Filser (2002) : un décor, une intrigue (l'histoire racontée) et une action (la relation entre l'objet, ici le produit sublimé par le chef, et le client). Néanmoins cette relation entre l'objet et le client peut également s'analyser comme une forme de prescription de la part du chef étoilé.

\section{Les formes de prescription du chef étoilé}

La prescription est l'influence qu'une ou plusieurs personnes exercent sur le comportement et le choix d'un ou plusieurs individus. Selon les cas, elle peut signifier recommandation, ordre ou précepte, mais n'est 
toujours qu'une proposition puisque l'individu à qui l'on fait une prescription a toujours le choix d'une action potentielle (Stenger et Coutant, 2009). Le rapport de prescription est établi lorsqu'il y a acceptation et conformation, même partielle, à une prescription. Cette acceptation conduit alors à valider les savoirs de l'autre (Hatchuel, 1994, cité par Stenger et Coutant, 2009). Le prescripteur n'est pas forcément un intermédiaire marchand mais peut être un tiers. Deux types de prescription sont susceptibles d'influencer le processus d'achat: la prescription technique, qui comble des lacunes de l'acheteur sur l'étendue des possibles d'un produit en termes d'usages, par exemple, ou la prescription de jugement qui fournit non seulement des informations supplémentaires mais aussi des appréciations - Hatchuel (1995) cite l'exemple des étoiles du guide Michelin.

Dans le cas d'un restaurant et d'une cuisine mettant en avant les produits locaux, il y aurait à la fois une prescription technique, lorsque le chef propose une manière inédite de préparer un produit local, et une prescription de jugement, dans la mesure où le choix de tel ou tel producteur pour des fromages présentés sur la carte, par exemple, est une forme d'appréciation de celui-ci. Le nom du producteur inscrit sur la carte est une autre illustration d'une prescription de jugement. Celle-ci peut également intervenir lors d'échanges avec le personnel en salle, quand il répond aux questions de clients sur la provenance, voire sur la fabrication des produits proposés sur la carte. Dans ce dernier cas, on peut parler d'une forme de coprescription, puisque le client participe activement, par ses questions, à l'expression de ses attentes (la connaissance du produit local, voire l'achat ultérieur) et met tout en œuvre pour leurs réalisations (Stenger et Coutant, 2009).

Ces situations de prescriptions, qu'elles soient techniques ou de jugement, ont-elles une influence sur le processus d'achat de produits locaux par les clients de restaurants étoilés ? Nous avons cherché à mieux appréhender les relations qui peuvent exister entre l'expérience gastronomique de produits locaux par un individu dans le cadre d'un repas dans un établissement étoilé et son comportement d'achat ultérieur de ces mêmes produits chez les producteurs.

\section{LES PRODUITS LOCAUX}

\section{SUBLIMÉS PAR LES CHEFS :}

UNE RÉPONSE AUX ATTENTES DES

CLIENTS MAIS UN RÔLE RELATIF SUR

LEUR COMPORTEMENT D'ACHAT

Une enquête qualitative, de type exploratoire, a été entreprise afin de mieux cerner le rôle effectif des chefs étoilés dans l'achat de produits locaux à travers l'expérience de gastronomie vécue par leurs clients. L'analyse des entretiens réalisés confirme les travaux de Merle et Piotrowski (2012) sur les raisons qui amènent les individus à consommer des produits locaux. Par ailleurs, les récits des expériences vécues dans des restaurants étoilés permettent d'avancer quelques éléments d'explications sur l'influence que peut avoir un chef étoilé sur le comportement d'achat de produits locaux de ses clients.

\section{Méthodologie de recherche}

Selon Évrard, Pras et Roux (2003), l'entretien individuel peut être une meilleure solution que le focus group pour le nombre d'idées émises. Nous avons donc eu recours à des entretiens semi-directifs. Pour la constitution de notre échantillon de convenance, nous nous sommes attachés à interroger des personnes ayant déjà vécu une ou plusieurs expériences dans des restaurants étoilés. Douze entretiens, de trente à quarante-cinq minutes, ont été réalisés auprès de clients de restaurants étoilés, qu'ils soient résidents du lieu de l'établissement ou touristes. La plupart d'entre eux ont donc évoqué des expériences gastronomiques vécues en Savoie et Haute-Savoie, c'est-àdire à proximité de leur domicile, mais également des expériences vécues dans d'autres régions, sur leur lieu de vacances. Il s'agit, pour l'essentiel, d'expériences dans des établissements classés une étoile par le guide Michelin (Le Lamartine ou Le Bateau ivre, au Bourget-du-Lac ; Le Clos des sens, à Annecy; La Tête d'oie, à Lyon ; Le Michel Sarran ou L'Amphitryon, à Toulouse), plus rarement dans des restaurants classés deux étoiles ou plus (Les Morainières, à Jongieu ; l'établissement de Marc Veyrat, en son temps, et aujourd'hui celui de son élève, Yohann Comte, à Veyrier-du-Lac). 
Tableau I • Structure de l'échantillon de type qualitatif

\begin{tabular}{lcccc}
\hline Prénom & Sexe & Âge & Profession, CSP & Durée de l'entretien \\
Fabienne & Féminin & 40 ans & Secrétaire administrative & $31 \mathrm{~min}$ \\
Romain & Masculin & 27 ans & Cadre & $35 \mathrm{~min}$ \\
Philippe & Masculin & 40 ans & Directeur régional & $38 \mathrm{~min}$ \\
Catherine & Féminin & 51 ans & Assistante & $28 \mathrm{~min}$ \\
Géraldine & Féminin & 39 ans & Secrétaire de direction & $30 \mathrm{~min}$ \\
Anne & Féminin & 37 ans & Mère au foyer & $27 \mathrm{~min}$ \\
Yann & Masculin & 31 ans & Cadre & $36 \mathrm{~min}$ \\
Élisabeth & Féminin & 44 ans & Enseignante & $25 \mathrm{~min}$ \\
Olivier & Masculin & 40 ans & Enseignant & $28 \mathrm{~min}$ \\
& & & & \\
\hline
\end{tabular}

Nous considérons que les personnes constituant notre échantillon sont des clients "non experts", pour ce qui est de leurs expériences gastronomiques. En effet, même si les personnes interviewées ont eu plusieurs expériences dans des restaurants étoilés (entre deux et sept), les récits mettent en avant le caractère exceptionnel de l'événement et non la description d'un bon repas. Nous reprenons ici la distinction de Quan et Wang (2004) qui soulignent que, plus les clients sont novices, plus l'expérience de repas est décrite avec une forte intensité des émotions ; à l'inverse, les clients plus experts décrivent l'expérience vécue comment un moment agréable, un bon repas. La teneur de nos entretiens tend à confirmer le caractère novice des personnes interrogées. Le niveau d'implication concernant les produits locaux des individus interrogés est, quant à lui, plutôt élevé, puisque tous en consomment plus ou moins régulièrement. On peut donc supposer un certain niveau de connais- sance et, peut-être, un certain niveau d'exigence vis-à-vis des produits locaux consommés, plus spécifiquement lors d'expériences gastronomiques chez des étoilés.

Les participants à ces entretiens ont été interviewés à leur domicile ou sur leur lieu de travail. Les profils sont diversifiés en termes d'âge et de sexe (cf. tableau 1) ; tous résident en Rhône-Alpes (Savoie, HauteSavoie, Rhône). Concernant la taille de notre échantillon, nous avons observé le critère de saturation sémantique en termes de contenu des réponses obtenues.

Un guide d'entretien a été utilisé ; il est découpé en trois parties, de la plus générale à la plus spécifique ( $c f$. encadré 1). La première s'attache à mieux comprendre les habitudes et pratiques de l'individu concernant la gastronomie, en général, la consommation de produits locaux et la fréquentation de restaurants étoilés, en particulier. Une seconde partie cherche à mieux appréhender l'expérience recherchée par l'individu lorsqu'il se rend dans un établissement étoilé, et ses attentes vis-à-vis des produits locaux qui y sont proposés. Enfin, la troisième partie du guide d'entretien se focalise sur les comportements d'achat et de recherche des produits locaux, à la suite de l'expérience dans un restaurant étoilé : de quelle manière une expérience vécue dans un restaurant gastronomique peut-elle susciter un achat de produits locaux chez un producteur? quels moyens sont alors mis en œuvre ?

Les entretiens, d'une durée moyenne de trente-cinq minutes, se sont déroulés en face à face. Ils ont été intégralement retranscrits. Pour mener à bien cette recherche qualitative, qui se situe dans une approche d'approfondissement et de compréhension, une analyse de contenu thématique a été privilégiée, apparaissant comme la plus adaptée pour faire ressortir la richesse des discours collectés (Évrard, Pras et Roux, 2003). Nous avons donc réalisé cette analyse par la construction de thèmes a posteriori en dégageant des dissemblances et ressemblances dans les verbatim. Pour cela, nous avons suivi un procédé de comparaison- généralisation menant à des catégories, puis nous avons repéré de thèmes redondants ayant un sens par rapport à la question de recherche et à sa résolution.

\section{Résultats}

- Des individus intéressés par la gastronomie en général et par les produits locaux en particulier. Les personnes interrogées ont exprimé 
un intérêt marqué pour la gastronomie et la cuisine d'une manière générale. Cela se traduit par l'achat de revues et de livres, par du temps libre consacré à la cuisine, mais également par l'intérêt accordé aux produits alimentaires, et notamment aux produits locaux. Les raisons de consommation sont variées mais on retrouve dans les réponses les éléments soulignés par Merle et Piotrowski (20/2) : l'achat et la consommation de produits locaux sont une façon de consommer responsable et de respecter les saisons, un moyen de s'assurer de la fraîcheur et du goût des produits, ou encore de créer du lien social et de faire vivre l'économie locale. C'est aussi une manière de faire connaître les richesses culinaires aux "étrangers" (Dion, Sitz et Rémy, 20 10). C'est enfin un moyen de s'intégrer dans une région dont on n'est pas originaire et, ainsi, de se faire adopter par les locaux (cf. encadré 2 ). Si ces résultats permettent de mieux comprendre la manière et les raisons pour lesquelles les individus achètent et consomment des produits locaux, ils fournissent également des explications à l'importance qu'ils accordent à trouver ces mêmes produits sur les plus grandes tables.

Car on peut supposer que les personnes s'offrant une expérience gastronomique sont, en règle générale, des personnes ayant un intérêt pour l'alimentation et, donc, qu'elles accordent une certaine importance à l'origine et à la qualité des produits. Les produits locaux semblent être une réponse à ces exigences de qua-

\section{Encadré | • Guide d'entretien}

\section{Trois temps}

I. Mieux comprendre les habitudes et pratiques de l'individu par rapport à la gastronomie en général (intérêt particulier), les produits locaux (en consomme-t-il ?) et aux restaurants étoilés en particulier (y va-t-il régulièrement ou non, si oui, se situent-ils dans sa région d'origine ou pas ?)

2. Mieux appréhender l'expérience recherchée par l'individu lorsqu'il se rend chez un étoilé, d'une part (surprise, variété...), et comprendre ce qu'il recherche lorsqu'il achète des produits locaux, d'autre part (revivre une expérience, qualité de l'aliment...). Si achat de produits locaux : quels types de produits sont privilégiés (labels) ? quels les produits emblématiques de son souvenir d'expérience ? où les trouvent-ils ? fait-il la démarche de les chercher?

3. Se focaliser sur les comportements d'achat et de consommation de ces produits, en relation avec une expérience chez un étoilé : l'expérience chez ce dernier a-t-il ou pas suscité l'achat?

\section{Questions}

I. Est-ce que la gastronomie vous intéresse ? Comment cela se traduit-il ? (restaurants, ateliers, revues...)

2. Allez-vous régulièrement/occasionnellement/exceptionnellement chez un étoilé ?

3. Si oui, sont-ils dans votre région ou non?

4. Dans quels types d'étoilés allez-vous? (plutôt Ferran Adrià ou plutôt Bocuse)

5. Pourquoi? Qu'y recherchez-vous?

6. À travers vos expériences, pouvez-vous dire que vous accordez une place importante à la connaissance des produits locaux?

7. Pourquoi cela est important pour vous?

8. Achetez-vous des produits locaux? Si oui, lesquels?

9. Lorsque vous êtes chez un étoilé, commandez-vous des plats à base de produits locaux?

10. Si oui, pouvez-vous me raconter les souvenirs que vous en gardez (au niveau de goût, de la présentation du plat...)

11. Qu'attendez-vous chez un chef étoilé du point de vue des produits locaux ?

12. Est-ce que vous attendez du chef étoilé qu'ils vous renseignent sur les produits utilisés? Aimeriez-vous plus d'informations sur les produits locaux?

13. Après une expérience chez un étoilé, est-ce que vous allez être tenté d'acheter les produits que vous avez découverts?

14. Pourquoi en achetez-vous?

15. Est-ce que vous faites ça dans votre région ou seulement quand vous êtes touriste?

16. Quels types de produits achetez-vous le plus fréquemment?

17. Comment faites-vous pour les trouver?

18. Êtes-vous content ou déçu de votre achat lors de la dégustation ? Pourquoi ? 


\section{Encadré $2 \cdot$ Les raisons de la consommation de produits locaux • Raisons invoquées}

\author{
Une manière de consommer responsable \\ "Pour moi c'est important, primordial. Pour une question \\ écologique d'abord" (Catherine). \\ "C'est pas un acte militant, mais ça coule de source. Mes parents \\ avaient le même mode de fonctionnement" (Yann). \\ "[...] mais j'avoue que c'est pas pour le goût super raffiné que je \\ vais le prendre là-bas. Je vais le prendre parce que c'est un \\ fromage local" (Élisabeth) \\ Respecter les saisons \\ "[...] et puis tu retrouves les achats saisonniers comme là tu vas \\ acheter du potiron. Tu vas pas acheter des tomates en hiver" \\ (Romain). \\ Faire vivre l'économie locale et créer du lien social \\ "Moi je vais acheter directement chez eux et ça fait marcher le \\ commerce local" (Olivier). \\ "Et puis, t'as une relation avec les producteurs. T'as des bons \\ plans en plus" (Romain).
}

Un moyen de s'assurer de la fraîcheur, du goût des produits et de réduire les risques sanitaires

"Quand c'est local, c'est acheté plus frais normalement et pour moi c'est important" (Isabelle).

"C'est carrément meilleur au niveau du goût" (Romain).

"Pour mes produits frais je vais vraiment vers la qualité. Je suis toujours dans du qualitatif donc la viande, les fruits et légumes, les fromages, je ne les prends pas en grande surface" (Géraldine). "Je préfere aller chercher le produit à l'origine, aller voir le fermier que d'aller au supermarché. Je pense que c'est meilleur quand même" (Fabienne).

Un moyen de s'intégrer dans une région ou de faire connaître sa région

"La première fois, que je les ai cuisiné [diots et crozets], c'était pour faire plaisir à des amis qui étaient d'ici et pour leur dire : "Je suis en capacité de cuisiner vos produits'. Et ils se sont régalés"

(Fabienne).

"Quand on reçoit des gens d'autres régions, on aime bien faire connaître" (Élisabeth).

\section{Encadré 3 • Les éléments recherchés lors d'une expérience gastronomique}

Une mise en scène

"Une ambiance, un cadre, des mets raffinés, soignés, bien

présentés" (Fabienne, 40 ans).

Des expériences sensorielles fortes

"Quelque chose comme une autre planète, un ailleurs plein de plaisirs, de délices, le plaisir gustatif' (Catherine).

De la nouveauté, de la surprise

"Je recherche quelque chose que je retrouverai nul par ailleurs. Des goûts, je veux être surpris par ce qu'il y a dans l'assiette, par l'originalité de ce qu'on va me présenter" (Philippe).
"C'est découvrir des mélanges de saveurs qu'on n'a pas forcément l'habitude de faire chez soi" (Fabienne).

Une histoire autour du produit

"Ça rajoute une touche d'authenticité au plat, en plus de ce que tu as écrit sur le menu. Tu dis: 'Tiens, là je suis en train de manger une pomme de terre qui vient de la vallée d'Abondance'. Ca te donne le sourire aux lèvres en fait. Le producteur tu le connais pas, t'iras pas vérifier si c'est vrai, mais le principe c'est de te dire qu'on te raconte une histoire sur le produit [...], tu sens que c'est quelque chose qui est ancré dans une petite histoire locale" (Romain). lité, dans la mesure où ils véhiculent une image d'authenticité. Dès lors, il nous a semblé logique de retrouver dans notre échantillon des individus sensibles au fait de découvrir ou de redécouvrir des produits locaux sur les grandes tables. Cet échantillon pourrait donc être perçu comme représentatif d'une frange de la population plutôt experte. Néanmoins, au travers des discours collectés, nous nous sommes rendus compte que le niveau de connaissance était souvent limité aux produits locaux issus de la région de résidence habituelle. Leur curiosité à découvrir, via une expérience gastronomique, un produit local d'une autre région 
est restée la même dans un contexte touristique. Il s'agit donc moins, selon nous, d'individus qui seraient experts en produits locaux que d'individus ayant des attentes fortes en la matière, parce qu'ils ont un intérêt pour l'alimentation.

Il est dès lors intéressant de déterminer si les expériences vécues dans le cadre d'un restaurant étoilé proche du lieu de résidence principale, alors que le client pourrait se sentir plus expert en matière de produits locaux, pouvaient déclencher un achat consécutif à l'expérience gastronomique.

- Les produits locaux au menu des plus grandes tables : une forme efficace de prescription ? Les récits des expériences vécues dans des restaurants étoilés soulignent ce qui est habituellement recherché dans ces établissements : une mise en scène, des expériences sensorielles fortes, ou encore de la nouveauté et de la surprise ( $c f$. encadré 3). Dans la plupart des établissements cités, si les produits locaux sont à l'honneur, la communication sur le produit local se fait avant tout par l'assiette et par le travail réalisé par le chef afin de sublimer le produit.

En effet, les personnes que nous avons interviewées n'ont pas, pour la plupart, de souvenirs d'une mise en avant du produit local par le personnel en salle. Les exceptions tiennent à la volonté du chef d'en faire un élément de différenciation - c'est le cas de Marc Veyrat ou de Yohann Comte, élève de ce dernier. Ici, les clients évoquent la mise en avant de l'origine du produit, mise en avant qui va jusqu'au nom du producteur.
Produire un discours autour du produit fait alors partie intégrante de l'expérience vécue par le client du restaurant gastronomique : "[...] ça rajoute une touche d'authenticité an plat, en plus de ce que tu as écrit sur le menu. Tu dis tiens, là je suis en train de manger une pomme de terre qui vient de la vallée d'Abondance. Ca te donne le sourire aux lèvres en fait. Le producteur tu le connais pas, t'iras pas vérifier si c'est vrai, mais le principe c'est de te dire qu'on te raconte une histoire sur le produit [...] tu sens que c'est quelque chose qui est ancré dans une petite histoire locale”. À travers ce verbatim, on comprend l'importance du récit dans la constitution d'une offre expérientielle. Le récit devient en complément indispensable du plat pour cet individu et contribue à rendre mémorable l'expérience vécue.

Pour autant, cette sublimation et cette mise en avant du produit local sur les grandes tables suffisent-elles à favoriser l'achat de produits locaux après avoir vécu une expérience gastronomique ? Les réponses sont, à ce stade de notre étude, plutôt partagées. Même pour des individus ayant un intérêt marqué pour les produits locaux, le restaurant étoilé n'est ni le lieu ni le moment pour chercher des “bons plans”. L'expérience gastronomique provoquant un état de flow psychologique, une absorption totale de l'individu dans ce qu'il vit (Csikszentmihályi, 1990), la demande d'information en vue d'un achat ultérieur ne vient pas à l'esprit. Pour certains, demander où trouver le produit ne servirait à rien, car ils seraient incapables de refaire le plat chez deux. Pour d'autres, cette absence d'échanges d'information tient au fait que la cuisine d'un chef étoilé contient une part de secret qui ne serait être violée. Dans l'esprit du client, la notoriété du chef crée une distance qui l'incite à garder le silence. Le manque de proximité entre le chef, le personnel et le client a été plusieurs fois évoqué. Il y a "des choses qui ne se font pas" dans les restaurants gastronomiques, et demander des précisions sur l'origine du produit ou le nom du producteur, en font partie. Le cadre, les rites et rituels, le protocole qui entourent le repas gastronomique favorisent la distance dans l'échange, une distance qui semble proportionnelle au nombre d'étoiles puisque, dans leurs discours, les individus ont exprimé une plus grande aisance dès lors qu'ils étaient dans un restaurant non étoilé ( $c f$. encadré 4$)$.

Néanmoins, certains produits semblent se prêter plus facilement à l'échange d'information. C'est notamment le cas des vins et des fromages, mais peut-être aussi plus généralement des produits transformés, non par le chef étoilé, mais par le producteur lui-même ( $c f$. encadré 5). On en revient à l'idée que l'aura du chef étoilé et de sa cuisine semblent freiner les clients dans leurs démarches de connaissances des produits locaux.

Près d'un tiers des personnes de notre échantillon ont pourtant émis le souhait de plus de dialogue ou de plus d'informations sur les produits. Pour certaines personnes, cette mise 
Encadré 4 • Freins à la demande d'information sur les produits locaux dans les restaurants étoilés

État psychologique de flow

"C'est le moment que je recherche, c'est pas chercher les bonnes adresses" (Bénédicte).

Incapacité ressentie à refaire le plat chez soi

"Je me sens pas les armes d'égaler de toute façon et donc ce n'est pas la peine de demander. On n'est pas au même niveau" (Élisabeth).

La distance perçue entre le client et le chef et sa cuisine "On a peut-être perdu, avec le nombre d'étoiles, la simplicité de l'échange, une certaine proximité" (Philippe).

'Je pense qu'il y a une certaine distance, le respect du secret quelque part. II y a un savoir-faire et le savoir 'où se servir', je dirais. Pour moi, ça fait partie de son secret, de l'énigme" (Jacqueline).

"Sur une cuisine étoilée, y'aura plus de secrets, de confidentialité. Ce qui est de moins en moins vrai, parce qu'un chef étoilé n'hésite plus à faire des bouquins, des émissions dans lesquelles il va donner des astuces, des secrets mais il révèle pas ses sources de producteurs. En fait, je pense que le chef est pas forcément comme ça, mais y'a le cadre qui alourdit..." (Philippe).

"On aura moins l'impression de poser une question indiscrète.

Ou alors de s'entendre dire: 'Ah non, je vous dis pas où je vais chercher mes produits"' (Bénédicte).

"Je pense que si un étoilé m'avait présenté un plat avec ce riz, je serais allé l'acheter. C'est des choses comme ça que j'attendrais chez un étoilé. Qu'on me dise qui est le producteur de ce fameux riz, comment on le fait, pourquoi il a justement ce goût qui vient des terres de Camargue, ça je pense que ça serait forcément bien dans un repas étoilé" (Yann).

\section{Encadré 5 - L'échange d'information et les attentes autour des produits locaux}

"Il y a eu un plateau de fromages absolument démentiel où j'ai goûté des fromages que j'avais jamais mangé et là, sur un, j'ai dit : 'Là, il faut que vous me disiez où je peux en acheter'. Ben il m'a dit : 'Voilà, ça vient de chez un tel'. Et le lendemain on est allé en acheter" (Yann).

"Au dessert ils te proposaient des petites madeleines cuisinées avec des petits pots de confitures locales. Et là quand tu vois un produit fini que le restaurateur a acheté, s'il me plaît, là je me dirais: 'Tiens, j'irai en acheter'. Car tu sais derrière que forcément t'as pas besoin d'un savoir-faire démesuré car c'est un produit fini. Et donc là j'aurais besoin de l'adresse. Et quand le serveur les a amenés, il a dit d'où ça venait" (Romain).

en avant du produit ou de la recette locale par le chef étoilé est une réelle attente, qu'elles soient dans leur région ou qu'elles deviennent touristes et cherchent à découvrir la région à travers la cuisine locale : "Quand tu vas à Marseille, en tant que touriste, tu t'attends à ce qu'il te serve une bouillabaisse avec des poissons de roche. Qu'il se serve du produit local. J'irai chez ce chef car il va me faire goûter quelque chose de chez lui".

- Des comportements différents selon que le client est local ou touriste? Enfin, pour les individus de notre échantillon, les achats de produits locaux sont réguliers, même en période de vacances. Pendant leur séjour, certaines personnes font leurs achats sur les marchés ce qui, pour elles, est une manière de visiter la région et, en discutant avec les gens sur les marchés, de mieux la connaître. Le comportement d'achat du produit local sur le lieu d'origine du produit est alors primordial (" $\mathrm{La}$ sancisse de Toulouse, je l'achète à
Toulouse, pas à Lyon"). Du fait d'une méconnaissance de la culture culinaire de la région visités, le risque de faire un mauvais achat est perçu comme plus élevé.

Chacun a alors sa stratégie pour limiter ce risque : si certains vont chercher le poisson au port directement chez le producteur, d'autres font une visite de fabrique de nougats avant d'en acheter sur le lieu même de production, ou préparent en amont leurs éventuels achats via des recherches sur internet, ou encore 
se renseignent une fois sur place en discutant avec des acteurs locaux, les propriétaires de chambres d'hôtes, notamment, mais également les personnes rencontrées au hasard des visites et haltes.

C'est souvent dans les restaurants non étoilés que les touristes obtiennent des informations sur le produit local. La "barrière" des étoiles n'existant pas, discuter avec le personnel ou le propriétaire du restaurant serait une manière de reconnaitre la qualité du plat mangé et un bon moyen d'obtenir des "bons plans" sur les lieux d'achat des produits. Le produit local devient alors une forme de souvenir de vacances, susceptible de procurer une certaine nostalgie, une manière de prolonger les vacances à travers le goût de l'aliment rapporté. L'achat procure un plaisir lié à la découverte d'un produit alimentaire jusque-là inconnu, plaisir qui se prolonge une fois de retour chez soi lorsqu'il est dégusté, même si parfois il n'a pas le même goût (Masset et Decrop, 20 I0).

À ce stade de l'étude, nos résultats soulignent de manière évidente le caractère expérientiel d'un repas chez un chef étoilé. Certains chefs ont fait de la mise en avant du produit local leur spécificité. Les sensations ne sont alors plus seulement gustatives. À travers la façon dont le produit est mis en avant, le client se crée ses propres images, s'approprie le produit et son identité, ce qui contribue à faire de l'expérience vécue une expérience extraordinaire.

Pour autant, l'expérience vécue à travers la dégustation n'apparaît pas comme un relais évident pour l'achat d'un produit local. Les réponses données soulignent que le cadre et le protocole qui entourent la dégustation, mais également la cuisine du chef étoilé, constituent des freins au dialogue avec le personnel en salle. Ces freins ne permettent pas de répondre à toutes les attentes de clients qui seraient intéressés par un achat ultérieur, et notamment à celles concernant l'histoire et la provenance du produit local.

\section{Discussion}

Analysé sous l'angle du courant expérientiel, le client d'un restaurant étoilé n'apparaît plus seulement comme un consommateur passif d'une expérience de consommation, mais comme quelqu'un qui peut devenir actif de sa propre expérience. En effet, si la dégustation des plats cantonne le client à un statut de consommateur de sensations et de plaisirs gustatifs, celui-ci devient coacteur de sa propre expérience lors des échanges qu'il peut avoir avec le personnel en salle en posant des questions sur les produits. L'expérience est alors vécue de manière holistique, mêlant tant des sensations et des émotions que des éléments d'ordre cognitif. Cette expérience peut se prolonger dans le temps par la rencontre avec un producteur et l'achat d'un produit local, consécutivement à cette expérience gustative vécue.

Nos résultats permettent de distinguer les comportements d'achat de produits locaux selon que l'indi- vidu est un "local”, c'est-à-dire résident habituel du lieu de l'établissement ou originaire de celui-ci, ou qu'il est un "étranger", un touriste. Loin de ses habitudes et de ses repères, l'individu devenu touriste met en place diverses stratégies pour assouvir son envie d'achat de produits locaux. Il est alors un "touriste culinaire”, privilégiant l'approche du lieu et de sa culture au travers des activités qui entourent l'alimentation, dont notamment l'achat de produits locaux. En effet, le tourisme culinaire consiste à "découvrir et explorer la culture et l'histoire d'un lieu à travers son alimentation et les activités qui lui sont liées par la création d'expériences mémorables" (Long, 1998, p. 186). Il est à la recherche d'une authenticité lui permettant de saisir "l'esprit du lieu" (Jacobs et Smits, 2007) à travers de multiples activités comme les visites de marchés locaux ou celles de festivals mettant à l'honneur un produit local $^{(3)}$. Cette recherche d'authenticité permet à l'individu de s'approprier "un lieu géographique et un idéal de vie traditionnel proche de la nature" (Potterie, 1994, cité par Camus, 2004). L'achat du produit local sur son lieu même de production semble une évidence pour la plupart des personnes interrogées. Le produit local devient alors un stéréotype de la région ou du pays : il remplit des fonctions liées à la mise en valeur personnelle et à l'expression du moi (Masset et Decrop, 20 I0).

Nous avons également souligné le fait que le chef étoilé émet, selon nous, une forme de prescription de 
jugement à travers sa cuisine en sublimant le ou les produits locaux. S'il choisit tel fromage ou tel ingrédient chez un producteur particulier, cela exprime le fait qu'il est celui qui détient le savoir et qu'il est en mesure d'apprécier les qualités organoleptiques et de fraîcheur du produit sélectionné. Ce produit local peut donc être un produit brut (une viande, un poisson, un légume) mais, le plus souvent, c'est un produit préalablement transformé par le producteur (un fromage, une confiture), comme le montre nos résultats. Cette sublimation répond à la fois aux préoccupations néophiles du mangeur, le chef laissant parler sa créativité et attisant la curiosité du gourmet, mais également à son côté néophobe, exprimé par la crainte de goûter un aliment nouveau (Fischler, 1990) : le fait que le produit ait été choisi par le chef est en soi un élément rassurant, d'autant que son plat peut être inspiré d'une recette déjà existante. Certains producteurs deviennent ainsi les fournisseurs attitrés de ces chefs, leur nom pouvant même parfois figurer sur la carte du restaurant dans l'énoncé du plat (Bonnain-Dulon et Brochot, 2008).

Pour autant, le moment de dégustation semble être un moment à part pour certaines personnes interrogées. Le cadre et le protocole qui entourent le repas chez un étoilé participent à rendre ce moment unique, dans lequel le client n'est pas dans le plaisir de la recherche du produit local, mais dans sa seule dégustation. Deux types de chefs étoilés peuvent être distingués : ceux qui mettent en avant les produits locaux à travers leur seule cuisine, d'une part, ceux qui, en outre, se positionnent en ambassadeurs des produits locaux de leur région, ajoutant à la mise en scène de leur cuisine une histoire du produit, de sa découverte ou du producteur, d'autre part. La rhétorique du chef devient alors un élément à part entière de l'expérience gustative vécue. Le plaisir n'est plus seulement gustatif ou lié à la surprise et à la découverte, mais s'enrichit du lieu d'un produit, de ses racines. C'est ce récit et ses échanges autour du produit qui, selon nous, fait passer le chef étoilé du statut d'ambassadeur à celui de prescripteur.

Les établissements non étoilés seraient, plus souvent que ceux étoilés, de réels prescripteurs, dans la mesure où l'échange semble plus libre pour les clients. L'expérience de consommation étant moins exceptionnelle, l'individu est moins immergé dans ce qu'il vit et, dès lors, son esprit devient plus disponible pour d'autres choses, et notamment la recherche de produits locaux.

\section{ConClusion}

Nous avons tenté d'apporter un éclairage sur la manière dont le chef étoilé devient acteur de la valorisation des produits de sa région auprès de ses clients, et comment il est susceptible d'influencer, ou non, un acte d'achat chez les producteurs. Si la mise en valeur des produits locaux est une attente réelle chez les personnes interrogées, il semble néanmoins que la sublima- tion du produit et le cadre qui entourent le repas tendent à freiner les échanges avec le personnel qui permettraient de créer de réelles situations de prescriptions. La perception par les clients de la préservation du secret de la source d'approvisionnement des chefs y contribue également. Pourtant, les recherches antérieures ont souligné l'importance que les individus accordent aujourd'hui aux produits locaux. Les produits locaux, lorsqu'ils figurent sur la carte d'un établissement étoilé, bénéficient d'une belle promotion, puisqu'ils vont $\mathrm{y}$ être dégustés après avoir été mis en valeur. En ce sens, les chefs étoilés participent au développement de la notoriété de ces produits.

Mais un essor passe aussi par une volonté des institutions touristiques locales et régionales. Celle-ci se traduit notamment par la création et la promotion de routes gourmandes dans certaines régions de France (Provence, Aubrac) et par la communication de marque - marque Savoie, marque Provence, Terres d'ici (pour la région grenobloise), Les Jardins du pays d'Aubagne, pour ne citer qu'elles. En définitive, si la plupart des chefs étoilés valorisent les produits de leur région, il semble qu'ils n'en soient pas pour autant de réels prescripteurs. Néanmoins, leur force est de marier ces "nourritures nostalgiques"(4) avec leur créativité. Les produits locaux, et les recettes qui y sont associées, ne sont pas seulement empreints d'un certain traditionalisme, synonyme de repli sur soi, mais bien d'une “identité 
dynamique" qui conjugue héritage et création (Coulon, 2008).

Ces conclusions nécessitent d'être approfondies afin de préciser les liens existants dans le triptyque clientchef étoilé-producteur local, en interrogeant cette fois-ci des chefs étoilés et des producteurs locaux amenés à approvisionner ces derniers. Cela permettrait de dégager des pistes de réflexion en matière d'axes de communication (comme, par exemple, l'affichage de partenariats par les producteurs locaux).

Il serait également intéressant d'interroger des personnes se rendant dans des restaurants gastronomiques mais ne consommant pas de manière régulière des produits locaux. Les résultats seraient alors peut-être différents puisque l'individu, en totale situation de découverte du produit local, pourrait être davantage enclin à poser des questions afin de se procurer a posteriori ledit produit.

Enfin, une autre piste de recherche consisterait à recueillir les témoignages d'individus ayant vécu une expérience gastronomique chez un étoilé disposant d'une boutique où les produits sont en vente. En effet, à la lumière des verbatim que nous avons recueillis, ce n'était ni le lieu ni le moment pour obtenir des bons plans sur les produits locaux, pour la majorité des individus interrogés. Il serait intéressant de mieux comprendre l'attitude des individus lorsque des produits sont proposés à la vente sur le lieu même de dégustation, dans la mesure où un certain nombre de variables individuelles, comme le niveau d'implication de l'individu pour les produits locaux, son enracinement dans la région ou encore sa propension à rechercher de la variété dans son alimentation sont susceptibles de jouer un rôle dans la relation entre l'expérience gastronomique vécue par ce dernier et l'achat chez un producteur local.

NOTES

( I) Ces composantes sont: l'absorption, le contrôle individuel, la joie, la valeur attribuée, le processus spontané et continu et la découverte de nouvelles perceptions et de nouveaux processus.

(2) En dix ans, le nombre de restaurants étoilés par le guide Michelin aurait augmenté de $15 \%$ (www.rtl.fr, 27 février 20।2) .

(3) Jacobs et Smits (2007) distinguent le touriste culinaire occasionnel, pour qui participer à des activités culinaires n'est pas la motivation première, du touriste culinaire proprement dit, qui choisit sa destination en fonction de l'intérêt culinaro-culturel qu'elle présente. Le touriste culinaire se distingue du touriste gastronomique qui organise son voyage autour d'un ou de quelques établissements de renommée internationale et qui souhaite vivre des expériences gustatives uniques dans un cadre d'excellence.

(4) Nous faisons bien évidemment référence à l'ouvrage d'Olivier Assouly (2004, p. 17) qui décrit les produits du terroir comme des nourritures nostalgiques qui "se fondent en autorité dans cette relation privilégiée au sol, dont la matérialité est aussitôt dépassée, voire sublimée, par le caractère respectueux des gestes de production et de transformation. La terre est auréolée de propriétés et de vertus que le travail du laboureur ou du viticulteur porte à leur comble".

\section{RÉFÉRENCES BIBLIOGRAPHIQUES}

Virginie AMILIEN, "Préface : à propos de produits locaux", Anthropology of food, n 4, 2005.

Éric J. Arnould et Linda L. Price, "River Magic. Extraordinary experience and the extended service encounter," Journal of Consumer Research, vol. 20, $n^{\circ} \mathrm{I}, 1993$.

Philippe Aurier, Fatiha Fort et Lucie SIRIEIX, "Exploring terroir product meanings for the consumer", Anthropology of food, $n^{\circ} 4,2005$.

Olivier Assouly, Les Nourritures nostalgiques. Essai sur le mythe du terroir, Actes Sud, 2004.

Philomène BAYET-ROBERT, Approche exploratoire de l'expérience de repas gastronomique - éléments mémorisés et familiarité client. Le cas des grands chefs Relais \& Châteaux, thèse en Sciences de gestion, Université de Lyon 3 Jean Moulin, 201 I.

Rolande BONNAIN-Dulon et Aline Brochot, "Haut lieu touristique, haute gastronomie, haute clientèle. Le tiercé gagnant de la Côte d'Azur?, dans Julia CSERGo et Jean-Pierre LEMASSON (dir.), Voyages en Gastronomies, coll. "Mutations", n²50, éd. Autrement, 2008.

Sandra Camus, "Proposition d'échelle de mesure de l'authenticité perçue d'un produit alimentaire, Recherche et applications en marketing, vol. 19, 4, 2004

Christian Coulon, "Des différentes manières de croquer le Périgord", dans Julia CSERGo et JeanPierre LEMASSON (dir.), Voyages en gastronomies, coll. "Mutations", n² 250, éd. Autrement, 2008.

Antonella CARÙ et Bernard Cova, " Expériences de consommation et marketing expérientiel", Revue française de gestion, vol. 3, n 162, 2006. Jean-Pierre CorbeAu et Jean-Pierre POULAIN, Penser l'alimentation. Entre imaginaire et rationalité, éd. Privat, 2001.

Mihály CsíkszentMIHÁLYI, Flow. The Psychology of Optimal Experience, Harper Perennial, 1990. 
Delphine DıON, Lionel SITZ et Éric RÉMY, "Le sentiment régional comme levier d'action marketing", Décisions Marketing, n 58, 20 I0. Yves Évrard, Bernard Pras et Élyette Roux, Market, études et recherches en marketing, $3^{e}$ édition, Dunod, 2003.

Anne-Emmanuelle FIAMOR, "Valorisation alimentaire et développement local. Analyse à partir d'un exemple drômois", Anthropology of food, $n^{\circ} 8,2011$.

Marc FILSER, "Éditorial. L'expérience de consommation : concepts, modèles et enjeux managériaux", Recherche et applications en marketing, vol. 23, n³, 2008.

Marc FILSER, "Le marketing de la production d'expérience : statut théorique et implications managériales", Décisions Marketing, $n^{\circ} 28$, 2002

Claude FISCHLER, L'Homnivore, éd. Odile Jacob, 1990.

Armand HATCHUEL, "Les marchés à prescripteurs", dans Annie Jacob et Hélène Vérin, L'Inscription sociale du marché, coll. "Logiques sociales", L'Harmattan, 1995.

Patrick Hetzel, "Vers une approche expérientielle de la haute cuisine française. Lorsque le marketing sensoriel rime avec construction du sens", Revue française de marketing, n 196, 2004.

Morris B. HOLBROOK (dir.), Consumer Value. A Framework for Analysis and Research, Routledge, 1999.

Hersch JACOBS et Florence SMITS, "Le tourisme culinaire : un fort marqueur territorial. L'exemple du Canada", Actes des $6^{e s}$ Rencontres de Mâcon "Tourismes et territoires", septembre 2007.

Dominique KRÉZIAK et Isabelle FrOCHOT, "Co-construction de l'expérience touristique. Les stratégies des touristes en stations de sport d'hiver", Décisions Marketing, n 64, 2011.

Lucy M. LoNG, "Culinary Tourism. A folkloric perspective on eating and otherness", Southem Folklore, vol. 55, n 3, 1998.

Jule MASSET et Alain DeCROP, "Tourisme et possessions spéciales : le cas des souvenirs de vacances", dans Alain DECROP (dir.), Le touriste consommateur, coll. "Les métiers du tourisme", De Boeck, 20 I0.

Rémi MenCAReLLI, "L'interaction lieu-objet comme conceptualisation de l'expérience vécue : proposition d'un modèle intégrateur",

Recherche et Applications en marketing, vol. 23, n³, 2008.

Aurélie Merle et Mathilde PIOTROWSKI, "Consommer des produits alimentaires locaux : comment et pourquoi ?", Décisions Marketing, $n^{\circ} 67,2012$.
Anne-Hélène Prigent-Simonin et Catherine Hérault-Fournier,

"The role of trust in the perception of the quality of local food products: with particular reference to direct relationships between producer and consumer", Anthropology of food, n 4, 2005.

Shuai QUAN et Ning Wang, "Towards a structural model of the tourist experience: an illustration from food experiences in tourism", Tourism Management, vol. 25, n 3, 2004.

Claire RoEDERER, L'expérience de consommation : exploration conceptuelle, méthodologique et stratégique, thèse de doctorat en sciences de gestion, université de Bourgogne, 2008.

Thomas STENGER et Alexandre COUTANT, "La prescription ordinaire de la consommation sur les réseaux socionumériques. De la sociabilité en ligne à la consommation ?", communication lors des $14^{\text {es }}$ Joumées de recherche en marketing de Bourgogne, Dijon, novembre 2009.

Gunnar VITTERSO et Virginie AMILIEN, "From tourist product to ordinary food? The role of rural tourism in development of local food and food heritage in Norway", Anthropology of food, n 8 , 2011.

Joëlle VANHAMME, "La relation surprise-ravissement revisitée à l'aune du marketing expérientiel", Recherche et applications en marketing, vol. 23, n³, 2008. 